\title{
Prescription of Benzodiazepines and Related Drugs in Patients with Mild Cognitive Deficits and Alzheimer's Disease
}

\author{
Authors

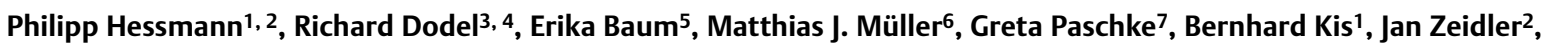 \\ Mike Klora², Jens-Peter Reese ${ }^{8}$, Monika Balzer-Geldsetzer ${ }^{3,4}$
}

\author{
Affiliations \\ 1 Department of Psychiatry and Psychotherapy, University \\ Medical Center Goettingen, Germany \\ 2 Center for Health Economics Research Hannover \\ (CHERH), Leibniz University Hannover, Germany \\ 3 Department of Neurology, Philipps-University Marburg, \\ Germany \\ 4 University Hospital Essen, Geriatric Centre Haus Berge, \\ Contilia $\mathrm{GmbH}$, Germany \\ 5 Department of General Practice, Philipps-University \\ Marburg, Germany \\ 6 Oberberg Clinics Berlin, Germany, and Faculty of \\ Medicine, Justus-Liebig-University Giessen, Germany \\ 7 Practice for General Medicine, Wiesbaden, Germany \\ 8 Coordinating Center for Clinical Trials, Philipps-University \\ Marburg, Germany
}

Key words

benzodiazepines, dementia, depression, neuropsychiatric specialists

received $\quad 13.11 .2017$

revised $\quad 17.12 .2017$

accepted $\quad 03.01 .2018$

\section{Bibliography}

DOI https://doi.org/10.1055/s-0044-100523

Published online: 31.1.2018

Pharmacopsychiatry 2019; 52: 84-91

(c) Georg Thieme Verlag KG Stuttgart · New York

ISSN 0176-3679

\section{Correspondence}

Philipp Hessmann, MD, MPH

Department of Psychiatry and Psychotherapy

University Medical Center Goettingen

Von-Siebold-Strasse 5

37075 Goettingen

Germany

p.hessmann@gmx.net

\section{ABSTRACT}

Introduction Benzodiazepines and related drugs (BZDR) should be avoided in patients with cognitive impairment. We evaluated the relationship between a BZDR treatment and the health status of patients with Alzheimer's disease (AD).

Methods Cross-sectional study in 395 AD patients using bivariate and multiple logistic analyses to assess correlations between the prescription of BZDR and patients' characteristics (cognitive and functional capacity, health-related quality of life (HrQoL), neuropsychiatric symptoms).

Results BZDR were used in $12.4 \%(n=49)$ of all participants. In bivariate analyses, the prescription was associated with a lower HrQoL, a higher need of care, and the presence of anxiety. Multivariate models revealed a higher risk of BZDR treatment in patients with depression (OR 3.85, $95 \% \mathrm{Cl}: 1.45-$ 10.27). Community-dwelling participants and those treated by neurologists/psychiatrists had a lower risk of receiving BZDR (OR 0.33, $95 \% \mathrm{Cl}: 0.12-0.89$ and OR $0.16,95 \% \mathrm{Cl}: 0.07-$ $0.36)$.

Discussion The inappropriate use of BZDR conflicts with national and international guidelines. We suggest evaluating indications and treatment duration and improving the knowledge of alternative therapies in healthcare institutions.

\section{Introduction}

The medical and non-medical care for patients suffering from Alzheimer's disease (AD) poses an enormous burden on patients and their families and a tremendous challenge for the public health systems worldwide [1]. On the one hand, this is due to the devastating course of the disease, leading to a continuous decline in the patients' cognitive and functional capacity, a high need of care and a considerable rate of institutionalization in long-term care facilities. On the other hand, the prevalence of $A D$ is high and the future num- ber of affected patients is expected to rise due to demographic changes. In Germany, the incidence of dementia is about 200.000 patients per year with a total number of about 1.5 million people suffering from dementia [2]. Due to the considerable increase in the prevalence of dementia as a consequence of the global demographic development, the worldwide numbers of dementia patients are assumed to be more than 65 million in 2030 [3]. 
In patients suffering from dementia, behavioral and psychological symptoms (BPSD) often occur and central nervous agents (e. g. antipsychotics, antidepressants, anxiolytics, benzodiazepines, etc.) are frequently used for the symptomatic treatment of agitation, aggressive behavior, anxiety, depression, and sleep disturbances. However, the majority of randomized, placebo-controlled clinical trials did not reveal evidence for effects of benzodiazepines and related drugs (BZDR) on certain BPSD like sleep disturbances and agitation in dementia patients [4]. Though, the use of BZDR in dementia patients is correlated with an increased risk of sedation, cognitive decline, and falls as well as an elevated hazard of dependency on BZDR and adverse events such as agitation, restlessness, and delirium $[5,6]$. Generally, an enhanced sensitivity of benzodiazepine receptors and a prolonged half-life of BZDR have to be considered in advanced ages. Therefore, a medication with BZDR in dementia patients is only recommended for a short period of time and preferably with short-acting substances [7]. Additionally, a large number of publications explored the impact of a treatment with BZDR on the risk of dementia. However, it is controversially discussed whether a long-term use of BZDR causally raises the probability of dementia [8].

Despite the increased risk of adverse events and limited evidence for the efficacy of BZDR, earlier studies reported a relatively high prescription rate in dementia patients [9-11]. Though, the majority of earlier evaluations were based on claims data of health insurance companies or considered dementia patients in certain healthcare settings (e. g., only nursing home inhabitants or community-dwelling patients) [9-15]. In contrast, our study includes patients with $A D$ across all severity stages of dementia and from different residential sites. Additionally, this evaluation was based on primary data as assessed by means of standardized questionnaires. Therefore, the patients' health status (e. g., cognition, activities of daily living, neuropsychiatric symptoms) could be explicitly evaluated.

Our cross-sectional analysis aimed at evaluating the prescription rate of BZDR in a German cohort of patients with AD and mild cognitive deficits. We hypothesized that the treatment with BZDR was associated with the patients' clinical status and socio-demographic parameters such as residential and family status, need of care, and treatment by neuropsychiatric specialists, etc. In particular, we hypothesized that the use of BZDR was correlated with the patients' cognitive status, functional capacity, the presence and severity of BPSD (i.e., depression, anxiety), and health-related quality of life (HrQoL). To our best knowledge, this is the first primary data analysis in Germany assessing prescription patterns of BZDR among people suffering from AD with regards to the patients' socio-demographic and clinical characteristics.

\section{Methods}

\section{Study design and the patients' clinical status}

For this cross-sectional evaluation, data of 123 nursing home inhabitants and 272 community-dwelling patients with mild cognitive deficits and AD according to the diagnostic criteria of the $\mathrm{Na}$ tional Institute of Neurological and Communicative Disorders and Stroke and the Alzheimer's disease and Related Disorders Associa- tion (NINCDS-ADRDA) were analyzed [16]. The patients and their caregivers were consecutively recruited from inpatient and outpatient study centers in the region of Marburg-Biedenkopf in Germany. The participants were asked for their pharmaceutical treatment over a period of three months before the interview. The prescription of BZDR was evaluated by means of the respective ATC codes (Anatomical Therapeutic Chemical Classification System) as defined by the World Health Organization (WHO): N05BA (BZDR - anxiolytics), N05CD (BZDR - sedatives and hypnotics), and N05CF (zopiclone, zaleplon, zolpidem). For this study, the prevalent use of BZDR was defined as any reported prescription or use of any BZDR within three months prior to the interview. Therefore, the date of the first prescription of BZDR was assessed as well as the daily intake frequency and the dosage of each substance.

Prior to the initiation of the assessment, the study was approved by the ethics committee of the Philipps-University Marburg (Approval-No. 46/08) and the Landesärztekammer Hessen (MC 29/2009). All participants gave their written consent. If a patient was unable to provide written consent, her/his legal guardian decided for or against the patient's participation. This publication is predicated on an evaluation of AD patients' health status, quality of life, and the use of medical and non-medical resources as assessed in personal interviews. Further details on the study design and the standardized questionnaires used are available from an earlier publication [17].

In brief, the patients' cognitive capacity was assessed with the Mini-Mental State Examination (MMSE), resulting in the following classification of dementia severity: mild cognitive deficits (27-30 pts.), mild $A D$ (20-26 pts.), moderate $A D$ (10-19 pts.), and severe $A D(0-9$ pts.) [18]. The caregivers of community-dwelling patients rated the patients' functional capacity by means of the Alzheimer's Disease Cooperative Study-Activities of Daily Living questionnaire (ADCS-ADL) [19] as well as the presence and severity of behavioral and psychological symptoms (BPSD) with the Neuropsychiatric Inventory (NPI) [20]. To evaluate the occurrence of depressive syndromes, the Geriatric Depression Scale (GDS) was used as a selfassessment by the patients [21]. Finally, the patients' HrQoL was rated with the disease-specific Quality of Life-Alzheimer's Disease questionnaire (QoL-AD) and the generic EuroQol Instrument (EQ5 D Index and EQ VAS) [22, 23].

\section{Statistical analysis}

For the data entry, a FileMakerPro 9.0 database was used (FileMaker Inc., Santa Clara, USA). All statistical analyses were carried out using Microsoft Office Excel 2003 (Microsoft Corporation, Redmond, USA) and SPSS Version 22.0 (IBM SPSS Statistics, Armonk, USA). Prior to the data analysis, a significance level of $\alpha=0.05$ was set. The patients' socio-demographic and clinical data are presented as means with standard deviation (SD), median, minimum, maximum or as total numbers of cases and percentages. The distribution of the dependent variables was evaluated by means of the Kolmogorov-Smirnov test. Afterwards, differences between the indicated groups were analyzed with Mann-Whitney $U$ tests and Chi-Square tests. In addition to the bivariate analysis, a multiple logistic regression model was used to determine associations between the prescription of BZDR and the patients' socio-demographic and clinical characteristics. For this purpose, crude and ad- 
justed odds ratios (OR) with $95 \%$ confidence intervals (95\% Cl) were determined and the Hosmer-Lemeshow test was applied to evaluate the goodness of fit for the regression model. Since the ADCSADL and the NPI were only applied in community-dwelling patients, both questionnaires were not considered for the multiple regression analysis. Concerning significant results in the bivariate analyses and the clinical relevance, the following independent variables were included after controlling for multicollinearity: age, gender (female, male), living situation (community-dwelling, nursing home), treatment by neurologists or psychiatrists (yes, no), level of care (three categories), disease severity (MMSE: four categories), presence of depression (GDS score), and HrQoL (EQ-5D Index, QoL$A D)$.

\section{Results}

Among the 395 study participants, 49 patients (12.4\%) were treated with BZDR at least once over a period of three months before the interview. Of these, the majority were women $(71.4 \%, n=35)$ similar to the proportion of female patients among those not receiving BZDR $(67.6 \%, n=234 ; p=0.593)$. On average, BZDR users were not significantly older than non-users $(80.0 \pm 9.3$, median: 84.0 vs. $78.7 \pm 8.5$, median: $80.0 ; p=0.119$ ). With regards to the respective substances, the following were most often prescribed: lorazepam (42.9\%, $n=21$; half-life: 9-19h), oxazepam (22.4\%, $n=11$; half-life: $4-15 h)$, diazepam (14.3\%, $n=7$; half-life: $30-56 h)$, and zopiclone (12.2\%, $n=6$; half-life: $2-6 \mathrm{~h}$ ).

As depicted in > Fig. 1, patients treated with BZDR significantly differed from those not medicated with BZDR regarding their resi- dential status. BZDR were more often prescribed in nursing home inhabitants compared to community-dwelling participants $(p<0.001)$. In total, BZDR were used in $22.0 \%(n=27)$ of the nursing home inhabitants compared to $8.1 \%(n=22)$ of the patients living in their own home environment. Altogether, $66.2 \%(n=262)$ of all participants were seen by neurologists or psychiatrists during the study period. However, BZDR were significantly more often prescribed among dementia patients who were not treated by a neuropsychiatric specialist $(63.3 \%, n=31$ vs. $36.7 \%, n=18 ; p<0.001)$ (> Fig. 1).

Additionally, patients not living in a stable partnership (single, divorced, widowed) were at a higher risk of receiving BZDR $(p=0.022)$ as well as those who were in need of care according to the German classification into three levels of care $(p=0.037)$ ( $\triangleright$ Fig. 1). Totally, $8.2 \%(n=15)$ of the patients not in need of care were medicated with BZDR compared to $16.1 \%(n=34)$ of patients who obtained professional care, with the highest proportion among those at level II $(20.3 \%, n=15)$. Furthermore, those participants not medicated with BZDR had received professional care for $3.2 \pm 3.7$ years on average (median: 2.0 ) compared to $5.1 \pm 4.9$ years (median: 3.0 ) among BZDR users $(p=0.016)$. In contrast, the disease duration was not significantly associated with the prescription of BZDR ( $\triangleright$ Table 1 ).

As detailed in > Table 1, the patients' cognitive capacity was similar in both groups, whereas the proportion of participants with mild cognitive deficits (MMSE 27-30) and mild dementia (MMSE 20-26) was slightly higher among BZDR users. A higher proportion of patients treated with BZDR showed depressive syndromes according to the results of the GDS than those not medicated with
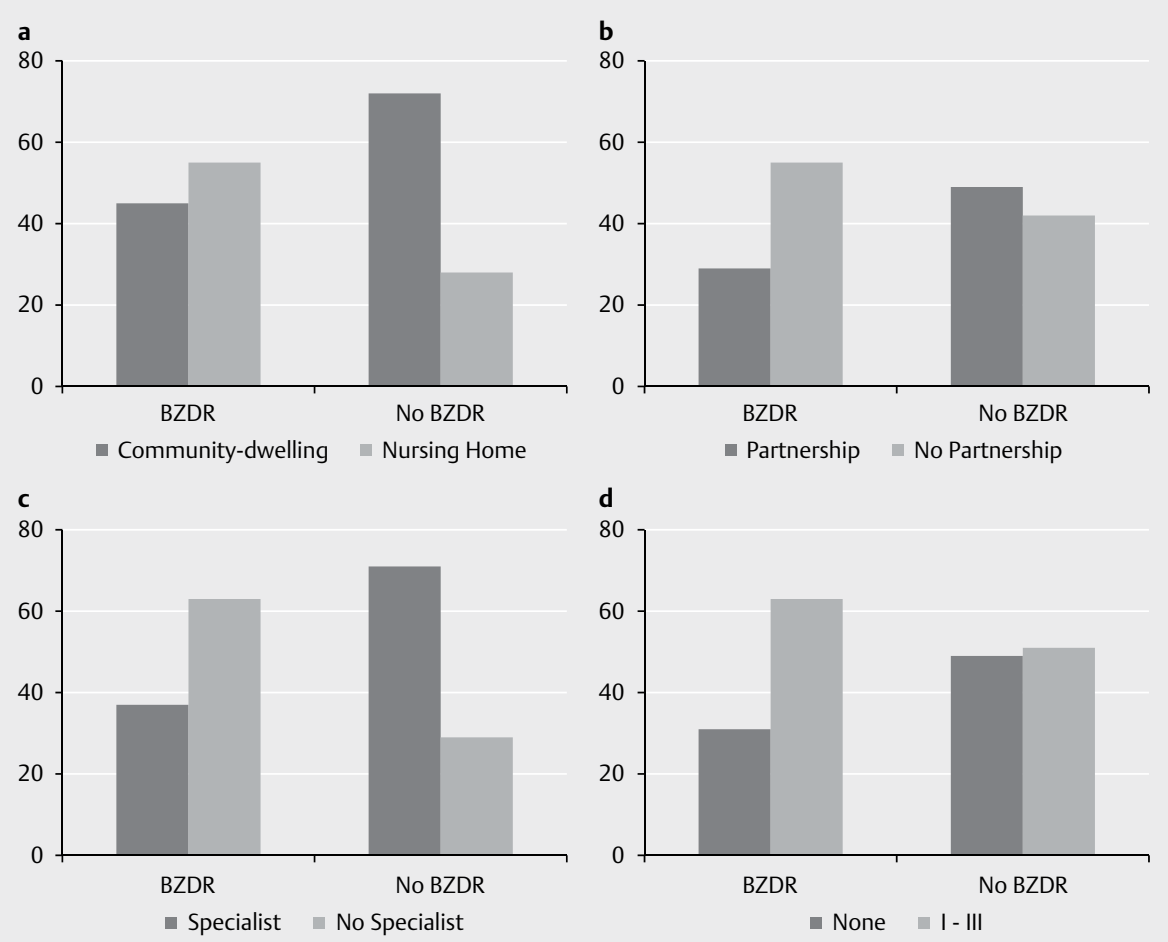

- Fig. 1 Prescription of benzodiazepines and related drugs (BZDR) according to the patients' socio-demographic characteristics. a Residential status. b Family status. c Treatment by neuropsychiatric specialists. $\mathbf{d}$ Level of care. 
- Table 1 Clinical and functional status of participants treated with benzodiazepines and related drugs (BZDR) compared to those not medicated with BZDR.

\begin{tabular}{|l|c|c|c|}
\hline & BZDR & No BZDR & p-value \\
\hline $\begin{array}{l}\text { Disease duration } \\
\text { (years) }\end{array}$ & & & 0.471 \\
\hline Mean \pm SD & $5.0 \pm 3.6$ & $4.7 \pm 4.0$ & \\
\hline Median (range) & $4.5(0-14)$ & $4.0(0-24)$ & \\
\hline MMSE & & & 0.311 \\
\hline Mean \pm SD & $14.9 \pm 8.8$ & $16.3 \pm 9.2$ & \\
\hline Median (range) & $15.0(0-29)$ & $19.0(0-30)$ & \\
\hline MMSE, $\mathbf{n}$ (\%) & & & 0.209 \\
\hline $27-30$ pts. & $15(30.6)$ & $94(27.2)$ & \\
\hline $20-26$ pts. & $18(36.7)$ & $87(25.1)$ & \\
\hline $10-19$ pts. & $12(24.5)$ & $118(34.1)$ & \\
\hline $0-9$ pts. & $4(8.2)$ & $47(13.6)$ & \\
\hline GDS & & & $<0.001$ \\
\hline Mean \pm SD & $13.6 \pm 5.3$ & $10.3 \pm 5.4$ & \\
\hline Median (range) & $13.0(3-25)$ & $9.0(0-27)$ & \\
\hline Depression (GDS) & & & $<0.001$ \\
\hline Probable (GDS $\geq 11)$ & $30(61.2)$ & $106(30.6)$ & \\
\hline $\begin{array}{l}\text { No Depression } \\
\text { (GDS } \leq 10)\end{array}$ & $11(22.4)$ & $155(44.8)$ & \\
\hline NPI total score & & & 0.188 \\
\hline Mean \pm SD & $22.8 \pm 17.6$ & $16.5 \pm 14.3$ & \\
\hline Median (range) & $21.0(1-59)$ & $12.0(1-65)$ & \\
\hline NPI distress score & & & \\
\hline Mean \pm SD & $11.0(3-28)$ & $7.5(0-28)$ & \\
\hline Median (range) & & & \\
\hline ADCS-ADL & $28.0(0-77)$ & $43.0(0-78)$ & \\
\hline Mean \pm SD & & & \\
\hline Median (range) & & & \\
\hline $\begin{array}{l}\text { All p-values based on chi-square test or Mann-Whitney U test. Due } \\
\text { to missing values, the percentages partly do not add up to } 100 \%\end{array}$ \\
\hline
\end{tabular}

BZDR (61.2\%, $n=30$ vs. $30.6 \%, n=106 ; p<0.001)$. Additionally, a higher proportion of patients who were treated with BZDR reported anxiety in the NPI $(77.8 \%, n=14$ vs. $31.2 \%, n=63, p<0.001)$. However, the frequency and severity of further BPSD, as measured with the NPI total score, was not significantly higher in participants medicated with BZDR as well as the extent of how intensively the caregivers perceived stress due to the patients' BPSD (NPI distress score). Furthermore, the participants' ability to perform activities of daily living, as evaluated with the ADCS-ADL, was low in the entire study population and patients treated with BZDR only showed insignificantly lower functional capacity than BZDR non-users ( Table 1).

-Fig. 2 illustrates the significant differences in the patients' HrQoL subject to the prescription of BZDR. The HrQoL was evaluated by means of the QoL-AD and the EuroQol Instrument as a selfassessment by the patients and as a proxy-rating by the caregivers. With regards to the QoL-AD and the EQ-5D Index, patients rated their HrQoL significantly higher than their caregivers (proxy assessment) in both, BZDR users and non-users. However, patients receiving BZDR reported significantly lower results in the QoL-AD than those not using BZDR in the self-assessment (27.5 \pm 5.2 , median: 28.0 vs. $30.7 \pm 5.6$, median: $30.5 ; p=0.001)$ and the caregivers' proxy-rating ( $21.4 \pm 5.1$, median: 21.0 vs. $24.6 \pm 7.2$, median: 24.0; $p=0.007$ ). Additionally, the self-reported results of the EQ-5D Index were considerably lower in patients using BZDR (0.513 \pm 0.365 , median: 0.499 vs. $0.690 \pm 0.313$, median: 0.813 ; $\mathrm{p}=0.002)$ as well as the proxy-evaluation according to the EQ-5D Index ( $0.264 \pm 0.355$, median: 0.110 vs. $0.476 \pm 0.360$, median: $0.400 ; p=0.001)$. In contrast, the EQ VAS did not reveal significant differences between the indicated groups, although BZDR treated participants reported lower average values in the self-report (55.2 \pm 17.5 , median: 50.0 vs. $61.1 \pm 19.7$, median: $50.5 ; p=0.111$ ) and the proxy-rating $(45.5 \pm 20.6$, median: 50.0 vs. $49.2 \pm 23.2$, median: 50.0; $p=0.432$ ).

As illustrated in $>$ Table 2 , the multiple logistic regression analysis showed that patients suffering from depression according to the results of the GDS were at a significantly higher risk of being treated with BZDR (OR 3.85, $95 \% \mathrm{Cl}: 1.45$ - 10.27). Additionally, patients living in their own home environment showed a considerably lower chance of receiving BZDR compared to nursing home inhabitants (OR 0.33, 95\% Cl: $0.12-0.89$ ). Finally, participants who were seen by neuropsychiatric specialists were medicated with BZDR less often than those patients not treated by neurologists and psychiatrists (OR 0.16, $95 \% \mathrm{Cl}: 0.07-0.36$ ) (॰ Table 2).

\section{Discussion}

In our study, about $12 \%$ of the participating dementia patients were medicated with BZDR at least once during the study period. These findings are largely in accordance with the prescription rates of earlier studies ranging from about $5 \%$ to $29 \%$ depending on the definition of BZDR use [12]. Additionally, earlier evaluations partly included patients with a certain residential status (community-dwelling vs. nursing home inhabitants) and suffering from dementia other than $A D$, complicating a direct comparison of the findings [9]. A longitudinal study by Montastruc et al. among 684 community-dwelling patients with mild to moderate AD living in France revealed that $8.5 \%$ of the participants were medicated with longacting BZDR. The authors concluded that the pathophysiological alterations in dementia patients and the pharmacological characteristics of the drugs are insufficiently considered when clinicians make decisions on the pharmaceutical treatment [24]. Rhee et al. found a comparable prescription rate of about $10 \%$ in 307 community-dwelling patients suffering from $A D$, vascular dementia, and other types of dementia [11].

However, some studies reported divergent prescription rates of BZDR. In an evaluation among 448 community-dwelling dementia patients in Germany, about $4 \%$ of the participants received BZDR [14]. In contrast, Goeman et al. found a significantly higher prescription rate of more than $31 \%$ in 412 Australian dementia patients and reported a medication with at least two BZDR at the same time in $4.5 \%$ of all the community-dwelling patients [10]. The authors assumed that BZDR are often used to symptomatically treat sleep disturbances and anxiety, even though evidence for a considerable effectiveness of a long-term treatment on those symptoms is lacking. Taipale et al. prospectively assessed the prescription of psychotropic drugs in more than 69.000 patients in Finland with the 
a

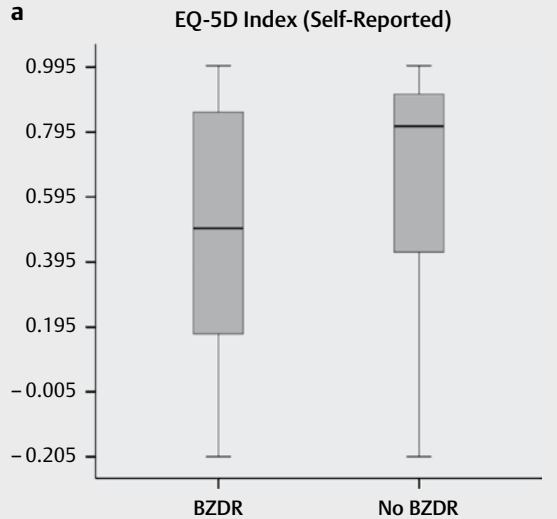

c

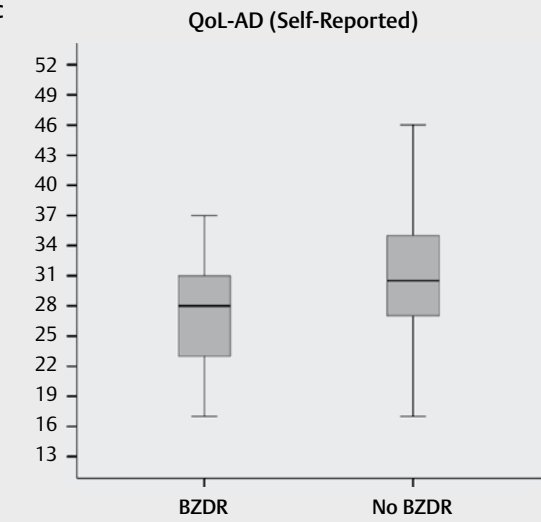

b

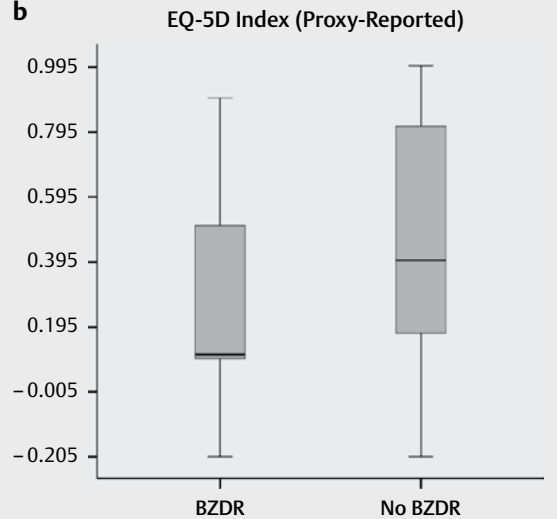

d

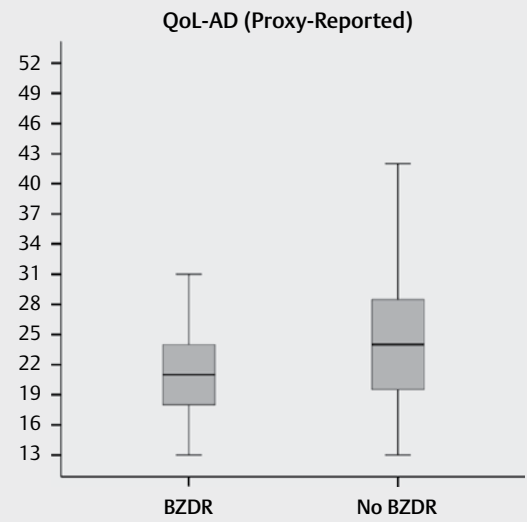

- Fig. 2 Self- and proxy-reported health-related Quality of Life according to the QoL-AD (a and $\mathbf{b}$ ) and the EQ-5D Index (c and $\mathbf{d}$ ) with respect to the prescription of benzodiazepines and related drugs (BZDR). EQ-5D: EuroQol - 5 Dimensions. QoL-AD: Quality of life - Alzheimer's disease questionnaire.

incident diagnosis of AD using administrative data. Over one year after the dementia diagnosis, $31 \%$ of the patients received BZDR, whereas the prescription rate decreased to $26 \%$ six years later. At this follow-up, the use of BZDR in patients with AD was comparable to those not suffering from dementia (25\%). Despite the high prescription rate of BZDR, the declining use might indicate a more cautious application of BZDR in the long-run [15].

Additionally, Taipale et al. found that $57 \%$ of all BZDR prescriptions were conducted by general practitioners, whereas $6 \%$ and $5 \%$ of BZDR were prescribed by neurologists and psychiatrists [15]. In our study, AD patients who were not seen by neurologists or psychiatrists showed a higher hazard of a treatment with BZDR. Nevertheless, about $37 \%$ of the patients medicated with BZDR were attending neuropsychiatric specialists. Potentially, these findings hint at an insufficient implementation of guideline recommendations in the healthcare facilities [25]. Therefore, the dissemination of recommendations for the therapy of dementia should be evaluated in further studies. Subsequently, effective strategies to implement the guidelines in the healthcare institutions (e. g. by integrating the recommendations in the quality management system) should be applied and the effect on the patients' treatment should be continuously evaluated. Additionally, the implementation of a standardized, inter-sectoral, and transparent documentation of the medication used in dementia patients might assist physicians in the avoidance of inappropriate medication such as BZDR [26]. Until now, evidence for the best treatment strategies for severe sleep disorders and anxiety in patients with dementia is lacking. Especially, antipsychotics are problematic due to their increased risk of morbidity and mortality [6].

Furthermore, our analyses revealed that patients living in longterm care facilities were at a higher risk of being treated with BZDR than community-dwelling participants ( $22.0 \%$ vs. $8.1 \%, p<0.001)$. These findings are similar to the results of a study by Huber et al., who reported a prescription rate of $17.3 \%$ for BZDR in 8.017 dementia patients living in German nursing homes on the basis of claims data [27]. Also, Jacob et al. described a higher likelihood for institutionalized patients to be medicated with BZDR considering the aggregated data of 61.713 dementia patients in Germany either treated by general practitioners or psychiatrists [28]. Vasudev et al. used claims data to longitudinally assess the dispensing of BZDR in Canadian dementia patients living in long-term care facilities. Thereof, $28 \%$ were medicated with BZDR in 2004, while the proportion of BZDR users decreased to $17 \%$ in 2013 [29]. Stevenson et al. reported a lower prescription rate of $13 \%$ for BZDR among 12.060 nursing home residents. However, the indication was inadequate in $42 \%$ of all cases and inhabitants suffering from dementia were at a higher risk of inappropriately receiving BZDR [30]. 
- Table 2 Multiple logistic regression analysis with the prescription of benzodiazepines and related drugs (BZDR) as dependent variable.

\begin{tabular}{|c|c|c|c|c|c|}
\hline Variable & $\begin{array}{c}\text { Regression coefficient } \\
(\log \text { OR) }\end{array}$ & Standard error & OR & $95 \%$ OR Cl & p-value \\
\hline Age & -0.02 & 0.03 & 0.98 & $0.38-0.98$ & 0.379 \\
\hline \multicolumn{6}{|l|}{ Gender } \\
\hline \multicolumn{6}{|l|}{ Female } \\
\hline Male & 0.02 & 0.47 & 1.02 & $0.40-2.58$ & 0.973 \\
\hline \multicolumn{6}{|l|}{ Living situation } \\
\hline \multicolumn{6}{|l|}{ Nursing home } \\
\hline Community-dwelling & -1.11 & 0.51 & 0.33 & $0.12-0.89$ & 0.028 \\
\hline \multicolumn{6}{|l|}{ Outpatient treatment } \\
\hline \multicolumn{6}{|l|}{ No specialist } \\
\hline Neuropsychiatric specialist & -1.85 & 0.43 & 0.16 & $0.07-0.36$ & $<0.001$ \\
\hline \multicolumn{6}{|l|}{ Care level, n (\%) } \\
\hline \multicolumn{6}{|l|}{ None (reference) } \\
\hline Level I & 0.74 & 0.55 & 2.10 & $0.72-6.12$ & 0.173 \\
\hline Level II or III & 0.85 & 0.69 & 2.34 & $0.60-9.12$ & 0.219 \\
\hline \multicolumn{6}{|l|}{ MMSE (pts.) } \\
\hline \multicolumn{6}{|l|}{ 27-30 (reference) } \\
\hline $20-26$ & -0.79 & 0.92 & 0.45 & $0.07-2.76$ & 0.390 \\
\hline $10-19$ & 0.04 & 0.71 & 1.04 & $0.26-4.21$ & 0.954 \\
\hline $0-9$ & -0.37 & 0.69 & 0.69 & $0.18-2.68$ & 0.591 \\
\hline \multicolumn{6}{|l|}{ GDS } \\
\hline \multicolumn{6}{|l|}{ No depression } \\
\hline Depression & 1.35 & 0.50 & 3.85 & $1.45-10.27$ & 0.007 \\
\hline QoL-AD score & -0.01 & 0.05 & 0.99 & $0.90-1.09$ & 0.884 \\
\hline EQ-5D index & 0.10 & 0.78 & 1.11 & $0.24-5.14$ & 0.895 \\
\hline
\end{tabular}

Moreover, patients using BZDR reported a significantly lower HrQoL with regards to the self-assessment and the proxy-rating of the QoL-AD and the EQ-5D Index in our study. Considering the single domains of the EQ-5D Index, patients medicated with BZDR showed significantly more often anxiety and a depressed mood in the self-assessment compared to BZDR non-users as shown in - Table 3. Additionally, BZDR were more often prescribed in patients suffering from depressive syndromes according to the GDS as well as those showing anxiety in the NPI. White et al. evaluated the management of BPSD among 230 dementia patients in an acute hospital setting, revealing that $12 \%$ of the patients were treated with BZDR, whereas the presence of anxiety was significantly associated with the use of BZDR [13]. These findings are similar to an evaluation by Maust et al. using the NPI to assess the presence of neuropsychiatric symptoms in dementia patients. The authors found that the degree of anxiety shown by the patients was significantly associated with the risk of being medicated with BZDR [31]. As we did not evaluate the indications for a medication with BZDR in our cohort, it remains unclear whether the prescription was due to the presence of depression and anxiety. Moreover, the continuity of a treatment with BZDR was not assessed. Certainly, knowledge of the initial symptoms leading to a prescription of BZDR and the duration of treatment is crucial as a discontinuation of the medication might cause withdrawal symptoms and rebound effects [32]. Therefore, further studies should evaluate the indications and the length of a BZDR treatment. However, a long-term use of BZDR, particularly with long-acting BZDR, is not in accordance with the current guidelines for the treatment of dementia patients, and a reduction of prescriptions should be strived for. Furthermore, the increased rate of BZDR use in patients fulfilling the diagnostic criteria of mild cognitive impairment or mild dementia is problematic, since this medication can cause a cognitive decline, subsequently complicating the differential diagnosis [33].

In conclusion, a proportion of about $12 \%$ of all participants in our study were medicated with BZDR. Especially, AD patients living in nursing homes, reporting depressive syndromes, anxiety, and a diminished HrQoL, as well as those not treated by neurologists or psychiatrists received BZDR significantly more often. Therefore, we suggest improving the awareness of risks of a treatment with BZDR in the healthcare facilities by providing evidence-based information on pharmaceutical therapy in dementia for patients, caregivers and clinicians. Additionally, a continuous evaluation of the implementation process of guideline recommendations should be established in order to diminish the prescription of BZDR.

Finally, our findings have to be interpreted under the consideration of several limitations. Due to the regionally restricted study design, the sample size, and the consecutive recruitment holding the risk of a selection bias, our study does not allow for a representative conclusion regarding the prescription prevalence of BZDR in Germany. Additionally, a recall bias has to be taken into consideration as the participants were asked for information on the pharmaceutical treatment over a period of three months prior to the interview. 
- Table 3 Self-reported problems according to the five EQ-5D dimensions in patients treated with BZDR compared to those not receiving BZDR.

\begin{tabular}{|c|c|c|c|c|}
\hline \multirow[t]{2}{*}{ EQ-5D dimensions } & Total & BZDR & No BZDR & \multirow[t]{2}{*}{ p-value } \\
\hline & n (\%) & n (\%) & n (\%) & \\
\hline Mobility & & & & 0.201 \\
\hline No problems $(0)$ & $131(33.2)$ & $15(30.6)$ & $116(33.5)$ & \\
\hline Any problems $(1+2)$ & $193(48.9)$ & $29(59.2)$ & $164(47.4)$ & \\
\hline Missing values & $71(18.0)$ & $5(10.2)$ & $66(19.1)$ & \\
\hline Self-care & & & & 0.022 \\
\hline No problems (0) & 209 (52.9) & $22(44.9)$ & $187(54.0)$ & \\
\hline Any problems $(1+2)$ & 114 (28.9) & $22(44.9)$ & $92(26.6)$ & \\
\hline Missing values & $72(18.2)$ & $5(10.2)$ & $67(19.4)$ & \\
\hline Usual activities & & & & 0.099 \\
\hline No problems (0) & $127(32.2)$ & $13(26.5)$ & $114(32.9)$ & \\
\hline Any problems $(1+2)$ & $196(49.6)$ & $31(63.3)$ & $165(47.7)$ & \\
\hline Missing values & $72(18.2)$ & $5(10.2)$ & $67(19.4)$ & \\
\hline Pain/discomfort & & & & 0.222 \\
\hline No problems (0) & $134(33.9)$ & $13(26.5)$ & $118(34.1)$ & \\
\hline Any problems (1 +2) & $189(47.8)$ & $31(63.3)$ & $161(46.5)$ & \\
\hline Missing values & $72(18.2)$ & $5(10.2)$ & $67(19.4)$ & \\
\hline Anxiety/depression & & & & 0.035 \\
\hline No problems $(0)$ & $140(35.4)$ & $13(26.5)$ & $127(36.7)$ & \\
\hline Any problems (1 + 2) & $183(46.3)$ & $31(63.3)$ & 152 (43.9) & \\
\hline Missing values & $72(18.2)$ & $5(10.2)$ & $67(19.4)$ & \\
\hline
\end{tabular}

Furthermore, the recruitment of patients with cognitive disorders other than AD cannot be fully excluded, because the diagnosis was primarily based on clinical diagnostic criteria (NINCDS-ADRDA). In addition, we used a cross-sectional study design not allowing for an assessment of the longitudinal use of BZDR in the cohort. However, other than the majority of earlier studies on the use of BZDR, we included community-dwelling and institutionalized patients with $A D$ across all severity stages of dementia and evaluated the patients' clinical status by means of standardized questionnaires.

\section{Acknowledgments}

This study was supported by an unrestricted grant from the Ernstand-Margot-Faber-Stiftung, Marburg, Germany.

\section{Conflict of interest}

All authors certify that they have no affiliations with or involvement in any organization or entity with any financial interest or non-financial interest in the subject matter or materials discussed in this manuscript.

\section{References}

[1] Prince M, Comas-Herrera A, Knapp M et al. World Alzheimer Report 2016: Improving healthcare for people living with dementia. Alzheimer's Disease International 2016; 6-20

[2] Ziegler U, Doblhammer G. Prevalence and incidence of dementia in Germany-a study based on data from the public sick funds in 2002. Gesundheitswesen 2009; 71: 281-290
[3] Prince M, Bryce R, Albanese E et al. The global prevalence of dementia: a systematic review and metaanalysis. Alzheimers Dement 2013; 9: 63-75

[4] Tampi R-R, Tampi D-J. Efficacy and tolerability of benzodiazepines for the treatment of behavioral and psychological symptoms of dementia: a systematic review of randomized controlled trials. Am J Alzheimers Dis Other Demen 2014; 29: 565-574

[5] Lapeyre-Mestre M. A review of adverse outcomes associated with psychoactive drug use in nursing home residents with dementia. Drugs Aging 2016; 33: 865-888

[6] Maust DT, Kim HM, Seyfried LS et al. Antipsychotics, other psychotropics, and the risk of death in patients with dementia: Number needed to harm. JAMA Psychiatry 2015; 72: 438-445

[7] Deuschl G, Maier W. 2016; S3-Leitlinie demenzen. In: Deutsche Gesellschaft für Neurologie (edt.) Leitlinien für Diagnostik und Therapie in der Neurologie. Online: www.dgn.org/leitlinien , last updated January 24, 2016 (accessed on October 10, 2017)

[8] Gray SL, Dublin S, Yu O et al Benzodiazepine use and risk of incident dementia or cognitive decline: prospective population based study. BMJ 2016; 352: i90

[9] Patel T, Slonim K, Lee L. Use of potentially inappropriate medications among ambulatory home-dwelling elderly patients with dementia: A review of the literature. Can Pharm J (Ott) 2017; 150: 169-183

[10] Goeman D, Harvey K, Lee C-Y et al. How Prolific is Psychotropic medicines use in people with dementia in australia within the community setting? A retrospective analysis. Drugs Real World Outcomes 2015; 2: 289-298

[11] Rhee Y, Csernansky J-G, Emanuel L-L et al. Psychotropic medication burden and factors associated with antipsychotic use: an analysis of a population-based sample of community-dwelling older persons with dementia. J Am Geriatr Soc 2011; 59: 2100-2107

[12] Tolppanen A-M, Voutilainen A, Taipale $\mathrm{H}$ et al. Regional changes in psychotropic use among Finnish persons with newly diagnosed Alzheimer's disease in 2005-2011. PloS One 2017; 12: e0173450 
[13] White N, Leurent B, Lord K et al. The management of behavioural and psychological symptoms of dementia in the acute general medical hospital: a longitudinal cohort study. Int J Geriatr Psychiatry 2017; 32: 297-305

[14] Wucherer D, Eichler T, Hertel J et al. Potentially inappropriate medication in community-dwelling primary care patients who were screened positive for dementia. J Alzheimers Dis 2017; 55: 691-701

[15] Taipale H, Koponen M, Tanskanen A et al. High prevalence of psychotropic drug use among persons with and without Alzheimer's disease in Finnish nationwide cohort. Eur Neuropsychopharmacol 2014; 24: 1729-1737

[16] Blacker D, Albert M-S, Bassett S-S et al. Reliability and validity of NINCDS-ADRDA criteria for Alzheimer's disease. The National Institute of Mental Health Genetics Initiative. Arch Neurol 1994; 51: 1198-1204

[17] Reese J-P, Hessmann P, Seeberg $G$ et al. Cost and care of patients with Alzheimer's disease: clinical predictors in German health care settings. J Alzheimers Dis 2011; 27: 723-736

[18] Folstein M-F, Folstein S-E, McHugh P-R. "Mini-mental state”. A practical method for grading the cognitive state of patients for the clinician. J Psychiatr Res 1975; 12: 189-198

[19] Galasko D, Bennett D, Sano M et al. An inventory to assess activities of daily living for clinical trials in Alzheimer's disease. The Alzheimer's Disease Cooperative Study. Alzheimer Dis Assoc Disord 1997; 11: 33-39

[20] Cummings J-L, Mega M, Gray K et al. The neuropsychiatric inventory: Comprehensive assessment of psychopathology in dementia. Neurology 1994; 44: 2308-2314

[21] Yesavage J-A, Brink T-L, Rose T-L et al. Development and validation of a geriatric depression screening scale: a preliminary report. J Psychiatr Res 1982; 17: 37-49

[22] Logsdon R-G, Gibbons L-E, McCurry S-M et al. Assessing quality of life in older adults with cognitive impairment. Psychosom Med 2002; 64: 510-519
[23] Brooks R. EuroQol: the current state of play. Health Policy 1996; 37 : 53-72

[24] Montastruc F, Gardette V, Cantet C et al. Potentially inappropriate medication use among patients with Alzheimer disease in the REAL.FR cohort: be aware of atropinic and benzodiazepine drugs!. Eur J Clin Pharmacol 2013; 69: 1589-1597

[25] Grol R, Grimshaw J. From best evidence to best practice: effective implementation of change in patients' care. Lancet 2003; 362: $1225-1230$

[26] Fiss T, Thyrian J-R, Fendrich $\mathrm{K}$ et al. Cognitive impairment in primary ambulatory health care: pharmacotherapy and the use of potentially inappropriate medicine. Int J Geriatr Psychiatry 2013; 28: 173-181

[27] Huber M, Kölzsch M, Rapp M-A et al. Antipsychotic drugs predominate in pharmacotherapy of nursing home residents with dementia. Pharmacopsychiatry 2012; 45: 182-188

[28] Jacob L, Bohlken J, Kostev K. Prescription patterns and drug costs in German patients with dementia in nursing homes and home-care settings. Int J Clin Pharmacol Ther 2017; 55: 9-15

[29] Vasudev A, Shariff S-Z, Liu K et al. Trends in psychotropic dispensing among older adults with dementia living in long-term care facilities: 2004-2013. Am J Geriatr Psychiatry 2015; 23: 1259-1269

[30] Stevenson D-G, Decker S-L, Dwyer L-L et al. Antipsychotic and benzodiazepine use among nursing home residents: findings from the 2004 National Nursing Home Survey. Am J Geriatr Psychiatry 2010; 18: 1078-1092

[31] Maust D-T, Langa K-M, Blow F-C et al. Psychotropic use and associated neuropsychiatric symptoms among patients with dementia in the USA. Int J Geriatr Psychiatry 2017; 32: 164-174

[32] Wolter DK. Discontinuation of benzodiazepines in old age: When and if so, how? Z Gerontol Geriatr 2017; 50: 115-122

[33] Airagnes G, Pelissolo A, Lavallée M et al. Benzodiazepine misuse in the elderly: Risk factors, consequences, and management. Curr Psychiatry Rep 2016; 18: 89 